\title{
Initial Matching of Multiple-View Images by Affine Approximation of Relative Distortions
}

\author{
Georgy Gimel'farb and Jian Quan Zhang \\ Centre for Image Technology and Robotics, \\ Department of Computer Science \\ Tamaki Campus, University of Auckland \\ Private Bag 92019, Auckland 1, New Zealand
}

\begin{abstract}
To match multiple views of a $3 \mathrm{D}$ scene, their relative geometric distortions have to be taken into account. We assume the disortions can be approximated by affine transformations. Images are matched by combining an exhaustive and directed unconstrained Hooke-Jeeves search for affine parameters, image pyramids being used to accelerate the search. The parameters found for several matches are statistically processed to relatively orient the images. Experiments with the RADIUS multiple-view images show a feasibility of this approach.
\end{abstract}

Keywords: multiple-view stereo, image matching, affine geometry

\section{Introduction}

3D scene reconstruction from multiple views captured by initially uncalibrated cameras merges cameras calibration and scene reconstruction into a single iterative process $4,6,7,11$. Usually views can be ordered so that neighbouring images cover almost the same part of the scene and have relative distortions of a known range (see, e.g., Figures [1 (3). To begin the process, each image pair has to be roughly oriented by matching corresponding points. The initial pairwise orientation allows, in principle, to roughly calibrate all the cameras. Then the rough calibration can be iteratively refined, along with the reconstruction of a $3 \mathrm{D}$ scene model.

In most cases, characteristic points-of-interest (POI) in the images such as corners [47] are matched. But generally the POI detection is not stable enough with respect to geometric and photometric image distortions as to ensure that the corresponding POIs can be simultaneously detected in different images. One may expect the POIs in one image allow to only select several subimages, or prototypes, to be matched to another image taking account of relative image distortions specified by a camera model.

\section{Affine vs. Projective Image Distortions}

If cameras are sufficiently far from a 3D scene, the projective pin-hole camera model can be approximated by the less complex affine model [5.9]. For simplicity, 

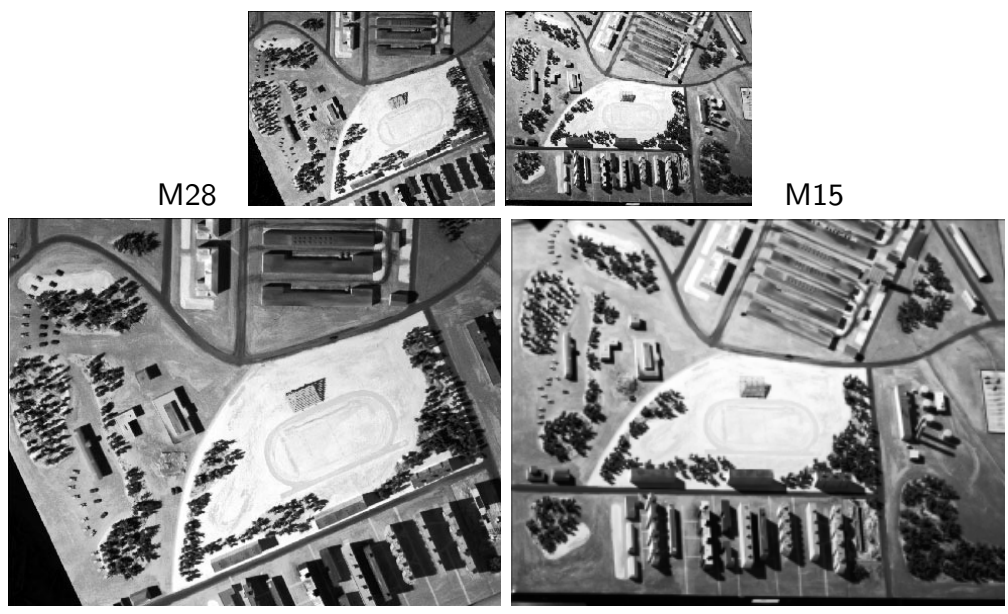

Fig. 1. The top and next-to-top levels of the M28-M15 image pyramids.

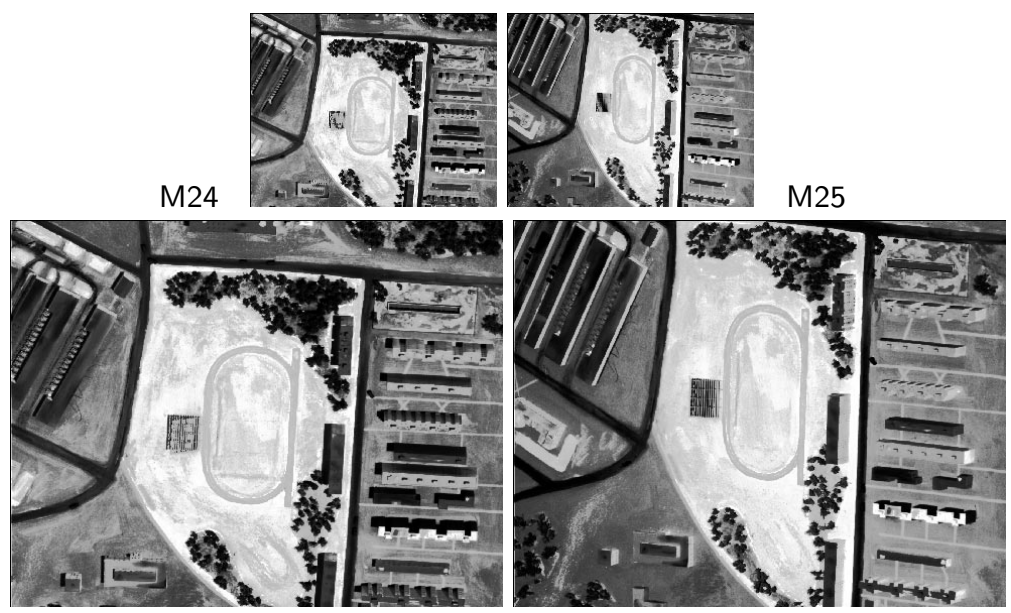

Fig. 2. The top and next-to-top levels of the M24-M25 image pyramids.

let the photometric image distortions be uniform and excluded by equalising the grey ranges of the images. Then the matching of a rectangular prototype $g_{1}$ to a quadrangular area in the image $g_{2}$ specified by the affine transformation can be guided by the mean square error (MSE) per pixel between the equalised grey values:

$$
D(\overline{\mathbf{a}})=\left[\frac{1}{|\mathbf{R}|} \sum_{\mathbf{i} \in \mathbf{R}}\left(g_{1}(\overline{\mathbf{i}})-g_{2}(\overline{\mathbf{f}}(\overline{\mathbf{i}} \mid \overline{\mathbf{a}}))\right)^{2}\right]^{\frac{1}{2}}
$$




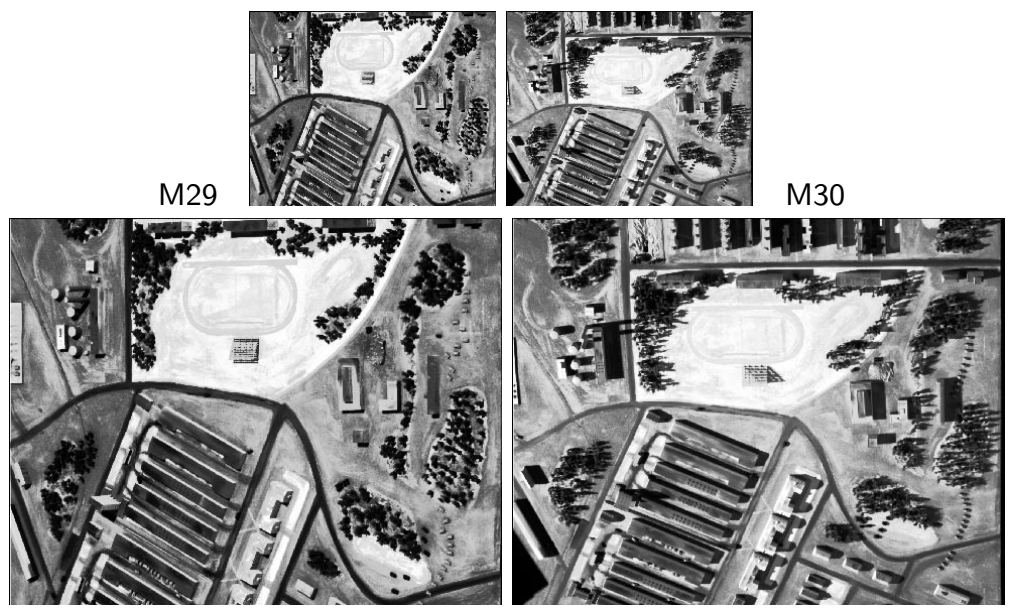

Fig. 3. The top and next-to-top levels of the M29-M30 image pyramids.

Here, $\mathbf{R}$ is a rectangular lattice supporting the prototype, and the pixel $\overline{\mathbf{i}}=(x, y)$ with the column $x$ and row $y$ coordinates in the prototype corresponds to the pixel $\overline{\mathbf{f}}(\overline{\mathbf{i}} \mid \overline{\mathbf{a}})=\left(x_{\overline{\mathbf{a}}}, y_{\overline{\mathbf{a}}}\right)$ in the image $g_{2}$, obtained by the affine transformation with the six affine parameters $\overline{\mathbf{a}}=\left[a_{1}, \ldots, a_{6}\right]: x_{\overline{\mathbf{a}}}=a_{1} x+a_{2} y+a_{3}$ and $y \overline{\mathbf{a}}=$ $a_{4} x+a_{5} y+a_{6}$. We assume the origin $(0,0)$ of the $(x, y)$-coordinates coincides with the lattice centre. Parameters $\left(a_{1}, a_{5}\right),\left(a_{2}, a_{4}\right)$, and $\left(a_{3}, a_{6}\right)$ specify, respectively, scales, shears, and shifts of the image $g_{2}$ with respect to the prototype. The grey level $g_{2}\left(x_{\overline{\mathbf{a}}}, y_{\overline{\mathbf{a}}}\right)$ can be found by a particular, e.g., nearest neighbour or bilinear interpolation of the image $g_{2}$.

Parameters â ensuring the best match, that is, the minimum MSE in Eq. (11) between the prototype $g_{1}$ and the transformed image $g_{2}$ :

$$
D(\hat{\mathbf{a}})=\min _{\overline{\mathbf{a}}} D(\overline{\mathbf{a}})
$$

can be used to form an initial estimate of the camera model for the image $g_{2}$ in relation to the known camera model for the image $g_{1}$. This rough relative model can then be used as the first approximation to begin the search for a projective model yielding the best matches.

\section{Combined Search for Affine Parameters}

The globally optimum match of Eq. (2) can be, in principle, found by exhausting all the possible geometric distortions $\overline{\mathbf{a}}$ of the image $g_{2}$. But the direct exhaustion of the six parameters for finding the minimum distance $D(\overline{\mathbf{a}})$ is not computationally feasible. More practical search (but for only a suboptimum match) is obtained by combining the exhaustion of a sparse grid of the relative shifts $a_{3}$ 
and $a_{6}$ with a direct Hooke-Jeeves unconstrained search [3] from every grid position for all the parameters $\overline{\mathbf{a}}$ that minimise the MSE.

The Hooke--Jeeves search starts with the initial parameter values $a_{1}=a_{5}=$ 1.0 and $a_{2}=a_{4}=0.0$ and iterates two steps: the exploration and the directional search within a given range $\left[a_{i, \min }, a_{i, \max }\right], i=1, \ldots, 6$, of the parameter values. At each exploration step, the value of a single parameter $a_{i}, i=1,2, \ldots, 6$, is changed with a given fixed increment $\pm \delta_{i}$ to find whether the value of the MSE $D(\overline{\mathbf{a}})$ can be decreased comparing to its current value, given the fixed values of all other parameters. If the MSE decreases, the incremented value is substituted for the initial one, and the next parameter, $i^{\prime}=2, \ldots, 6,1$, is explored until the MSE fails to decrease for all the parameters. Then the changes of the final parameters with respect to their starting values specify the possible direction of the MSE minimisation, and the directional search is performed by changing all the parameters simultaneously. These two steps are iterated at each grid position $t$ until the local minimum of the MSE, $D\left(\overline{\mathbf{a}}^{[t]}\right)$, is reached. The least value of $D\left(\overline{\mathbf{a}}^{[t]}\right)$ for the entire grid specifies the desired match. The obtained parameters $\overline{\mathbf{a}}^{[t]}$ are refined by repeating the Hooke-Jeeves search from the position of the prototype given by the found parameters $a_{3,[t]}$ and $a_{6,[t]}$.

Generally, the distance $D(\overline{\mathbf{a}})$ in Eq. (11) is a multi-modal function of the affine parameters $\overline{\mathbf{a}}$, and the search may stop far away from the desired best match. To partially overcome this drawback, we use a pyramidal image representation such as in Figures 1 - 3 to begin with the more stable low-resolution matching and refine the matching results at the higher-resolution levels of the pyramid.

POIs detected in $g_{1}$ at the lowest-resolution, or top level of the pyramid allow to select several large-size prototypes for matching to $g_{2}$ (see, e.g., Figures 4 6). Ideally, the affine parameters for a large-size prototype coincide with the matching parameters for the entire images. Therefore the statistically processed parameters found for the lowest-resolution prototypes can be used to relatively orient the images at the top level of the pyramids. Then these parameters are transferred to the higher-resolution levels for a subsequent refinement. In this case the scale and shear parameters preserve their values, and only the shift parameters should be changed according to the actual image resolution.

The above approach differs from the conventional matching with estimation of linear transformations [2] in that it does not linearize the MSE because the large-size prototypes are involved, and hence it does not build and use the normal equation matrix. The latter matrix usually is ill-conditioned because it depends on the image derivatives with respect to the affine parameters. Therefore these parameters are estimated more reliably by directly exploring the MSE to find a path to the minimimum instead of guiding the search by an analytic gradient.

\section{Experiments with the RADIUS Images}

Three image pairs M15-M28, M24-M25, and M29-M30 from the RADIUS-M set [8] selected for experiments are shown in Figures 10 [3. The next-to-top and 

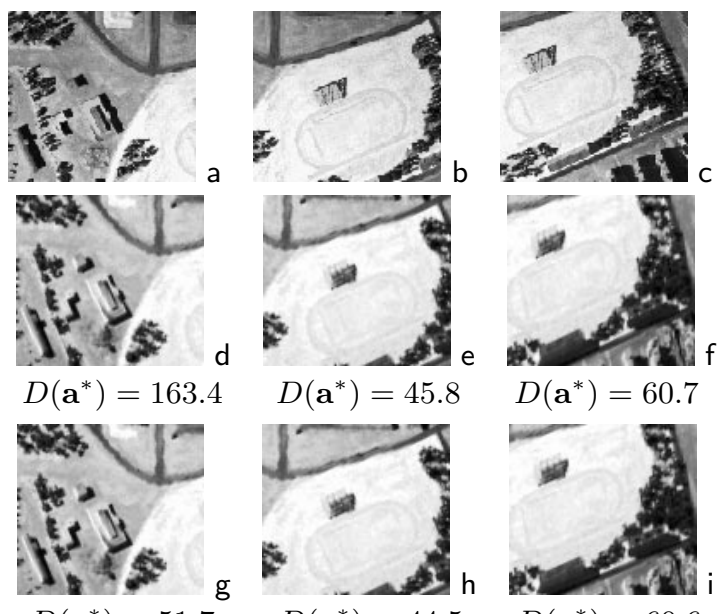

$D\left(\mathbf{a}^{*}\right)=45.8$

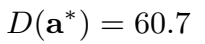

$D\left(\mathbf{a}^{*}\right)=51.7$
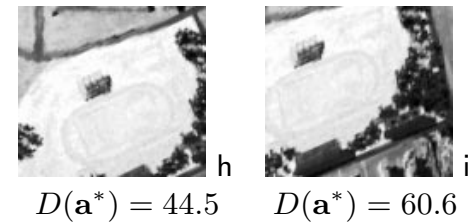

Fig. 4. M28 prototypes (a-c) and their initial (d-f) and refined (g-i) M15 matches; ranges of $a_{1}, a_{2}, a_{4}, a_{5}$ for g-i cases: [0.76,0.78], [-0.32,-0.10], [0.31,0.33], [0.68,0.72].
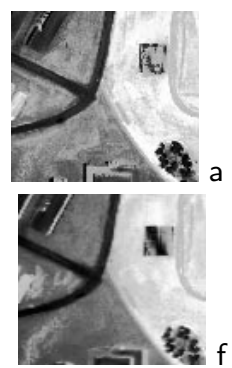

$D\left(\mathbf{a}^{*}\right)=36.5$

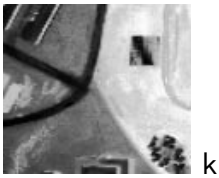

$D\left(\mathbf{a}^{*}\right)=36.0$
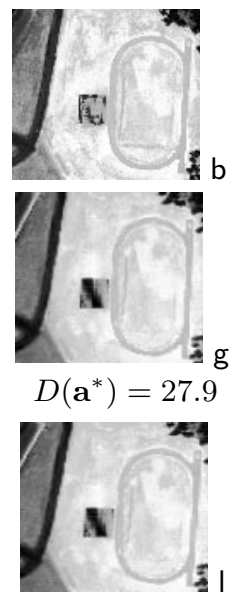

$D\left(\mathbf{a}^{*}\right)=26.2$
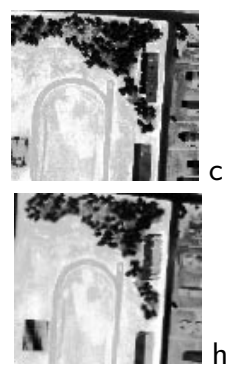

$D\left(\mathbf{a}^{*}\right)=59.1$

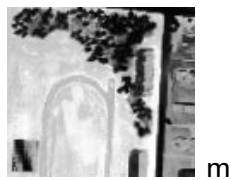

$D\left(\mathbf{a}^{*}\right)=60.0$
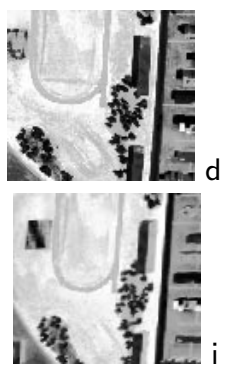

$D\left(\mathbf{a}^{*}\right)=67.4$

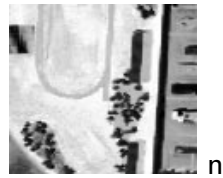

$D\left(\mathbf{a}^{*}\right)=45.4$
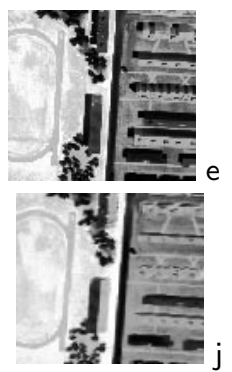

$D\left(\mathbf{a}^{*}\right)=50.6$

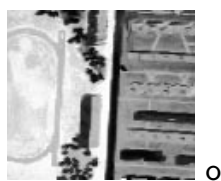

$D\left(\mathbf{a}^{*}\right)=49.1$

Fig. 5. M24 prototypes (a-e) and their initial $(\mathrm{f}-\mathrm{j})$ and refined (k-o) M25 matches; ranges of $a_{1}, a_{2}, a_{4}, a_{5}$ for k-o cases: [0.87,1.00], [0.00,0.02], [0.02,0.05], [1.02,1.12].

the top levels of the image pyramids are built by scaling the original images of size $1350 \times 1035$ down to $489 \times 384$ and $244 \times 192$ pixels.

We use only three-five prototypes per image pair, and the median values $\hat{\mathbf{a}}$ of the best-match parameters found for these prototypes specify the affine matrix $\hat{\mathbf{A}}$ for the relative image orientation and initial camera calibration. Each prototype 

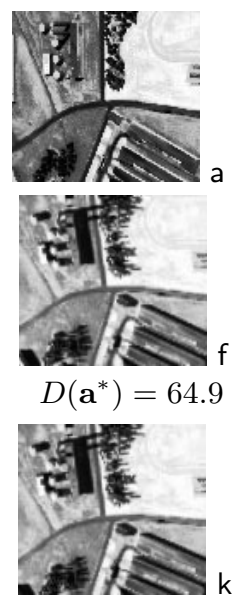

$D\left(\mathbf{a}^{*}\right)=64.2$

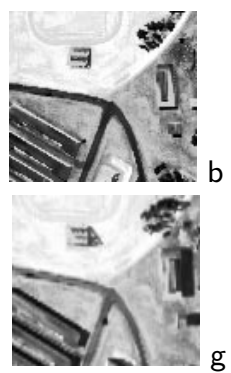

$D\left(\mathbf{a}^{*}\right)=53.6$

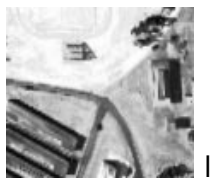

$D\left(\mathbf{a}^{*}\right)=52.4$
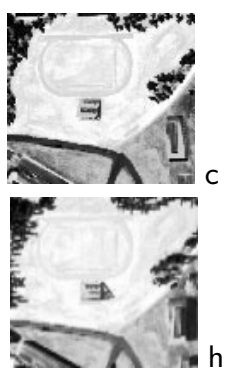

$D\left(\mathbf{a}^{*}\right)=51.4$

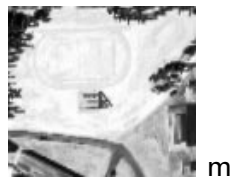

$D\left(\mathbf{a}^{*}\right)=50.7$
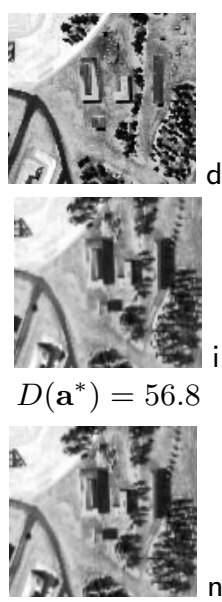

$D\left(\mathbf{a}^{*}\right)=56.4$

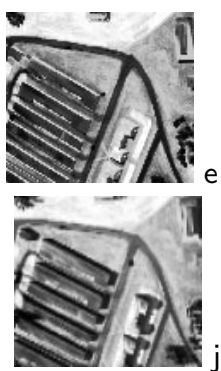

$D\left(\mathbf{a}^{*}\right)=66.7$

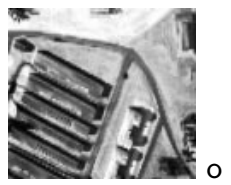

$D\left(\mathbf{a}^{*}\right)=65.6$

Fig. 6. M29 prototypes (a-e) and their initial ( $\mathrm{f}-\mathrm{j})$ and refined (k-o) M30 matches; ranges of $a_{1}, a_{2}, a_{4}, a_{5}$ for k-o cases: [0.98,1.02], [-0.02,0.02], [-0.04,0.04], [0.76,0.84].

covers about $20 \%$ of the image area and was rather arbitrary placed within the image provided several detected POIs are uniformly distributed within the prototype [10]. The prototypes of size $110 \times 100$ taken from M28, M24, and M29 at the top level of the pyramid and the initial and refined best matches of M15, M25, and M30 to these prototypes are shown in Figures 446.

Here, the sparse grid of shifts to be exhausted has steps of 15 pixels in both directions, and shifts of the grid origin within the square $15 \times 15$ did not effect the final match. Although the photometric distortions of the images are nonuniform, the median values of the refined affine parameters $\left[a_{1}, \ldots, a_{6}\right]$ for the best matches, namely,

$\begin{array}{lrrrrrr}\text { M28-M15: } & 0.78 & -0.30 & 154.9 & 0.33 & 0.70 & -124.0 \\ \text { M24-M25: } & 0.93 & 0.02 & 66.4 & 0.04 & 1.12 & -172.5 \\ \text { M29-M30: } & 1.00 & -0.20 & 11.1 & -0.02 & 0.80 & 145.5\end{array}$

reflect the basic geometric distortions of these images (here, the parameters $a_{3}$ and $a_{6}$ are scaled to the initial image resolution $1350 \times 1035$ pixels). The parameters vary within narrow limits indicated in Figures [46. The images M28, M24, and M29 relatively oriented with respect to the images M15, M25, and M30 using the above affine parameters, are presented in Figure 7 .

Refinement of the affine parameters at the next-to-top level of an image pyramids gives the similar results that also remain within narrow limits. Prototypes $200 \times 180$ taken from M28, M24, and M29 at the next-to-top level of the pyramid and the refined best matches of M15, M25, and M30 to these prototypes are shown in Figures 810 , The median values of the parameters $\left[a_{1}, \ldots, a_{6}\right]$ for 

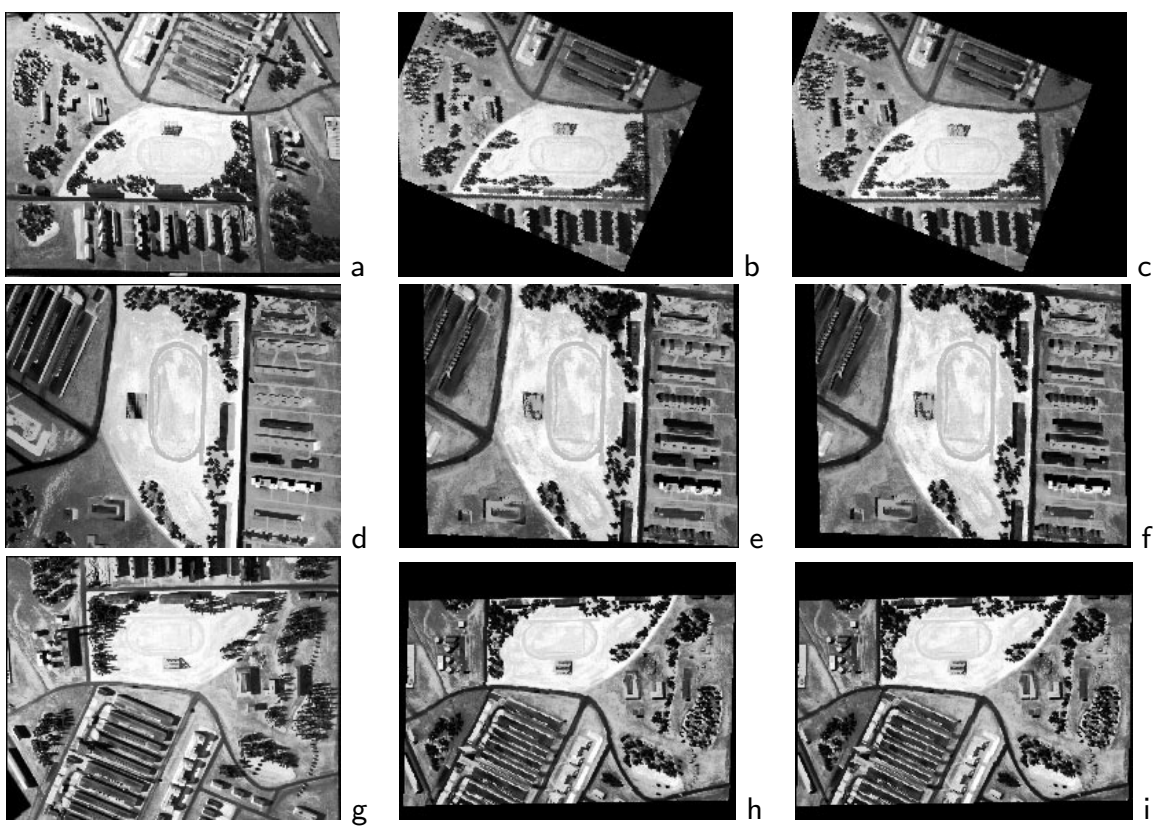

Fig. 7. Original images M15 (a), M25 (d), M30 (g) and the images M28 (b, c), M24 $(\mathrm{e}, \mathrm{f})$, M29 (h, i) relatively oriented using the refined affine parameters estimated, respectively, at the top and next-to-top levels of the image pyramids.

the best matches at this level are similar to the values found at the top level of the pyramids (see also Figure [7 c, f, i):

$\begin{array}{lrrrrrr}\text { M28-M15: } & 0.78 & -0.27 & 157.4 & 0.31 & 0.71 & -126.7 \\ \text { M24-M25: } & 0.92 & 0.03 & 63.5 & 0.03 & 1.11 & -172.5 \\ \text { M29-M30: } & 1.02 & -0.01 & 13.8 & -0.02 & 0.80 & 148.2\end{array}$

Figure 11] shows the overlaid original fields-of-view (FOV) of the M15 and M28 cameras in the 3D plane $Z=0$ with the $X$ - and $Y$-ranges $[-10,60]$ and $[-20,50]$, respectively, and the same FOVs after the affine transformation of the original projection matrix for the M28 camera so that the transformed projection matrix approximates the matrix for the M15 camera. Here, the affine parameters were estimated at the top level and the next-to-top level of the pyramid. Very similar results are obtained for the M24-M25 and M29-M30 cameras, too. Therefore the initial pairwise calibration of the cameras based on the affine distortion model seems to be sufficiently precise for the RADIUS images in spite of their non-uniform photometric distortions. 

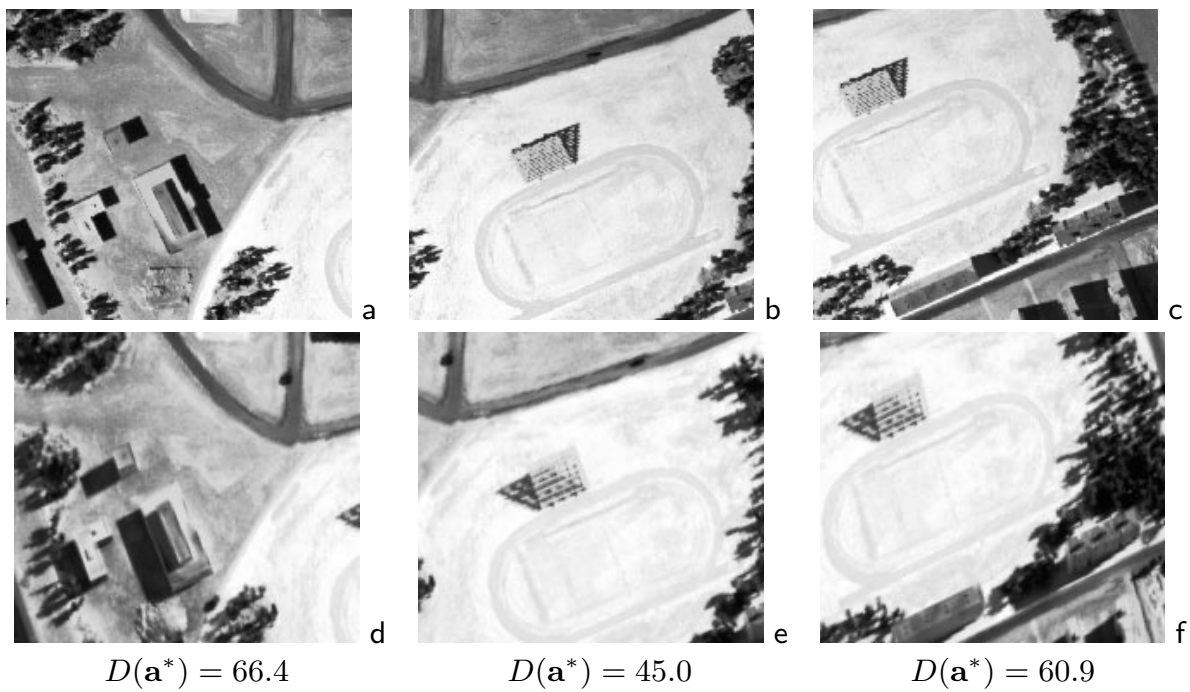

Fig. 8. Higher-resolution M28 prototypes (a-c) and their refined (d-f) M15 matches; ranges of $a_{1}, a_{2}, a_{4}, a_{5}$ for d-f cases: [0.77,0.79], [-0.27,-0.18], [0.31,0.34], [0.68,0.71].
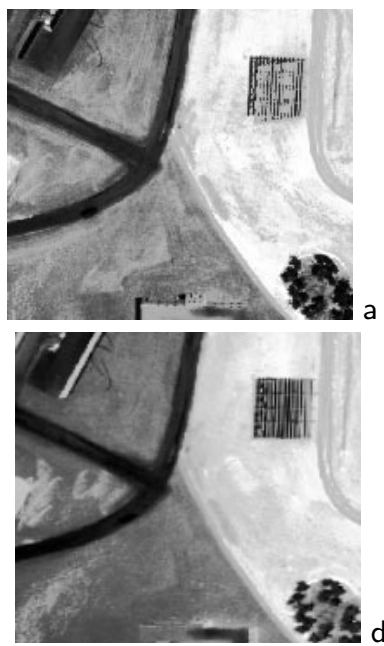

$D\left(\mathbf{a}^{*}\right)=34.5$
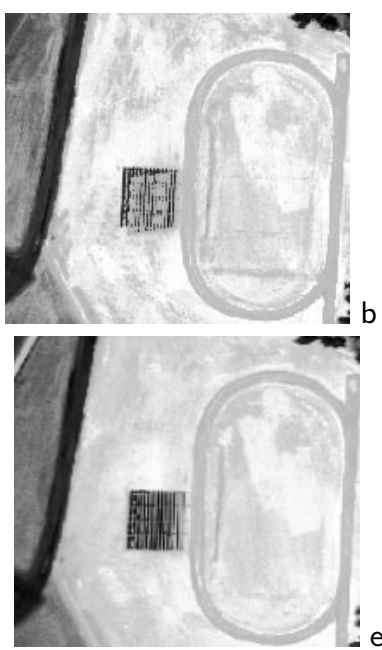

$D\left(\mathbf{a}^{*}\right)=7.9$
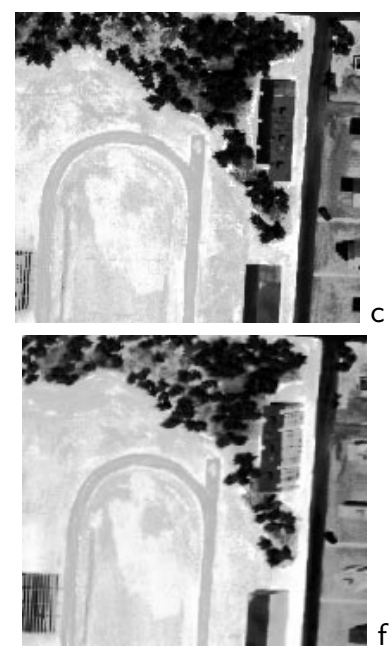

$D\left(\mathbf{a}^{*}\right)=48.3$

Fig. 9. Higher-resolution M24 prototypes (a-c) and their refined (d-f) M25 matches; ranges of $a_{1}, a_{2}, a_{4}, a_{5}$ for five matches: [0.87,0.97], [0.01,0.03], [0.03,0.06],[1.09,1.14]. 


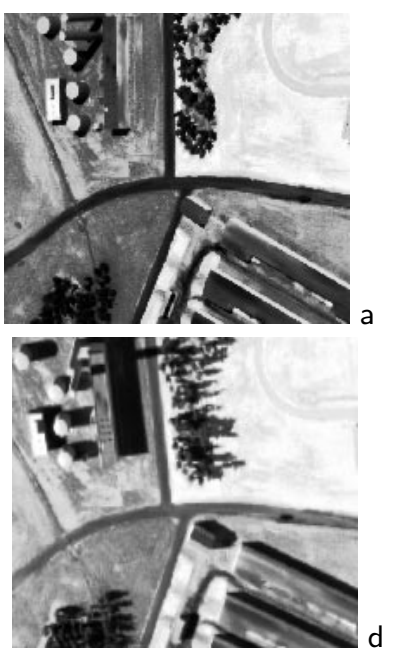

$D\left(\mathbf{a}^{*}\right)=66.3$

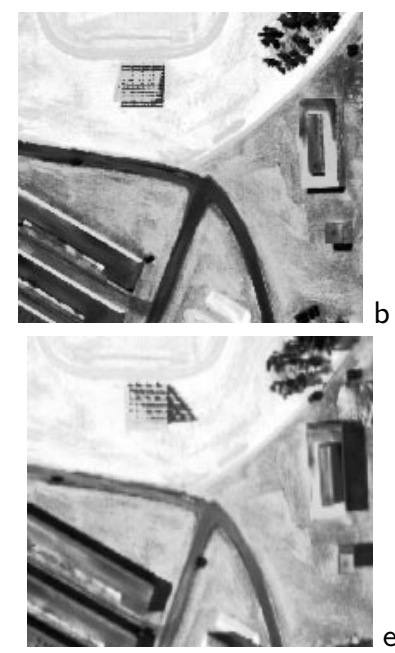

$D\left(\mathbf{a}^{*}\right)=51.0$

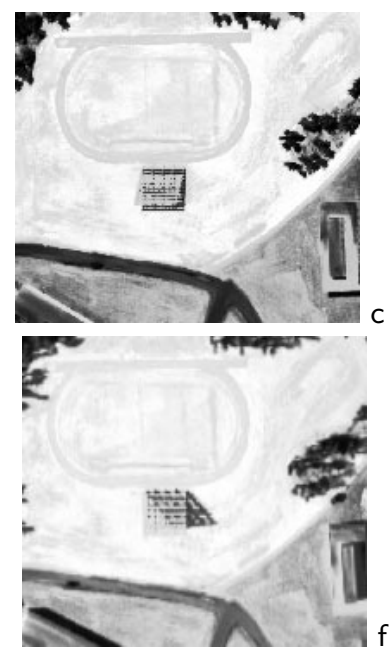

$D\left(\mathbf{a}^{*}\right)=42.4$

Fig. 10. Higher-resolution M29 prototypes (a-c) and their refined (d-f) M30 matches; ranges of $a_{1}, a_{2}, a_{4}, a_{5}$ for five matches: [0.98,1.04], [-0.04,0.01], [-0.05,0.01], [0.74,0.83].
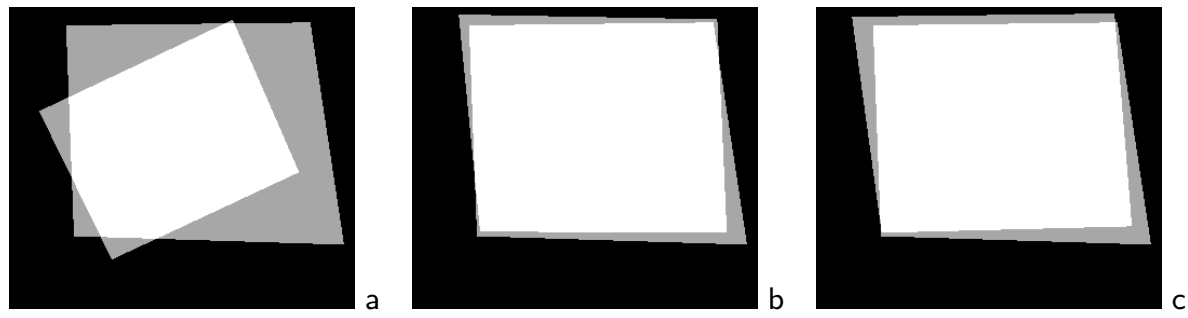

Fig. 11. Original FOVs for the M15 and M28 cameras (a) and the same FOVs after the affine transformation of the projection matrix for the M28 camera to the projection matrix for the M15 camera using the affine parameters estimated at the top level (b) and the next-to-top level (c) of the pyramid.

\section{Conclusions}

These and other experiments show that large-size areas in the multiple-view images of a 3D terrain can be in some cases successfully matched by combining the exhaustive and directed unconstrained Hooke-Jeeves search for the (locally) minimum MSE, provided the relative image distortions can be closely approximated by the affine transformation. In our case, the Hooke-Jeeves method is preferred over other popular numerical methods for unconstrained optimisation [1] (e.g., the Levenberg-Marquardt algorithm) because the Hooke-Jeeves one involves no analytically computed derivatives of the MSE in the parameter 
space. For our large-size image prototypes, the Jacobian matrix of the first derivatives of the MSE to be used in the Levenberg-Marquardt algorithm is usually ill-conditioned and too noisy to correctly guide the search.

The proposed approach to image matching has a moderate computational complexity so that in principle it can be used at the initial stage of the uncalibrated multiple-view terrain reconstruction. This approach permits us to simultaneously detect a small set of the initial corresponding points and form the relative affine camera models that roughly describe the pairwise image orientation. These models can then be used as the first approximation for estimating the relative projective camera models to begin the subsequent iterative process of refining the camera calibration and reconstructing the desired 3D scene model.

This approach exploits almost no prior information about a 3D scene, except for a range of the relative shifts between the corresponding points. But the images to be matched are assumed to contain a sufficient number of POIs for choosing the prototypes. Also, to make this approach practicable the problem of automatic choice of the appropriate prototypes, given a particular spatial scatter of the POIs, has to be solved.

\section{References}

1. Dennis J.E., Schnabel R.B.: Numerical Methods for Unconstrained Optimization and Nonlinear Equations. Prentice-Hall, Englewood Cliffs (1983)

2. Förstner, W.: Image matching. In: Haralick, R.M., Shapiro, L.G.: Computer and Robot Vision. Vol.2. Addison-Wesley, Reading (1993) 289-378

3. Himmelblau, D.M.: Applied Nonlinear Programming. McGraw-Hill Book, New York (1972)

4. Koch, R., van Gool, L. (eds.): 3D Structure from Multiple Images of Large-Scale Environments Lecture Notes in Computer Science, Vol. 1506. Springer-Verlag, Berlin Heidelberg New York (1998)

5. Koenderink, J., van Doorn, A.: Affine structure from motion. J. Optical Soc. America 8 (1991) 377-382

6. Maybank, S.J., Faugeras, O.: A theory of self-calibration of a moving camera. Int. J. Computer Vision 8 (1992) 123-152

7. Pollefeys, M., Koch, R., van Gool, L.: Self-calibration and metric reconstruction in spite of varying and unknown internal camera parameters. Int. J. Computer Vision 32 (1999) $7-25$

8. RADIUS model board imagery and ground truth (CD-ROM). Intelligent Machines Lab., University of Washington. Seattle (1996)

9. Shapiro, L.S.: Affine Analysis of Image Sequences. Cambridge University Press (1995)

10. Zhang, J.Q., Gimel'farb, G.: On detecting points-of-interest for relative orientation of stereo images. In: D.Pairman, H.North (eds.) Proc. Image and Vision Computing New Zealand 1999. Univ. of Canterbury, Christchurch, New Zealand, 30th-31st August 1999. Landcare Research, Lincoln (1999) 51-66

11. Zhang, Z., Deriche, R., Faugeras, O., Luong, Q.T.: A robust technique for matching two uncalibrated images through the recovery of the unknown epopolar geometry. Artificial Intelligence J. 78 (1995) 87-119 